\title{
A organização do streaming no Brasil: Telmex, Globo e a associação entre telecomunicações e audiovisual
}

\section{Joao Martins Ladeira}

\section{Resumo}

0 artigo investiga a estruturação do streaming no Brasil, analisando as interações entre dois dos principais agentes neste processo: Telmex e Globo. Analisa-se uma dimensão particular da renovação do audiovisual, com atenção à sua progressiva associação com tecnologias da informação. A institucionalização desta forma de acesso a conteúdo implica o uso de software, técnica para difundir material através de dispositivos dos mais diversos. Com ênfase na arqueologia da mídia, observa-se esta transição como parte de um cenário mais amplo. Na expectativa de compreender a lógica de definição deste objeto, relaciona-se este audiovisual dependente de software à trajetória pregressa da televisão. Como questão empírica, observa-se a dimensão histórica do vínculo entre Telmex e Globo, com atenção às múltiplas contradições contidas na rede instituída por ambas, no interesse de compreender como estes processos tomam parte na formulação dos sistemas contemporâneos de comunicação.

\section{Palavras-Chave}

Tecnologias da informação e comunicação. Estudos de televisão. Arqueologia da mídia.

Joao Martins Ladeira I joaomartinsladeira@gmail.com Doutor em Sociologia pelo Instituto Universitário de Pesquisas do Rio de Janeiro - IUPERJ, Brasil. Professor do Programa de PósGraduação em Ciências da Comunicação da Universidade do Vale do Rio dos Sinos - Unisinos, Brasil. Artigo financiado pelo CNPq (Humanidades, nº 470849/2014-2).

\section{Introdução}

Redes de fibra ótica em residências, garantindo acesso a conteúdo em ultra-alta definição, viável somente através de conexões em altíssima velocidade, a partir das quais se permite 0 consumo de audiovisual por meio de aplicativos operando em dispositivos móveis e outros instrumentos conectados. Cenário relevante na organização contemporânea do audiovisual, esta lógica redefine a própria concepção sobre o que seria televisão. Aqui, as experiências com imagem e som se desgarram em definitivo de um equipamento específico; da distribuição a partir os sistemas de broadcast; e, até mesmo, de sistemas segmentados a cabo ou satélite. Descrever este cenário aponta para um ambiente no qual as redes digitais para tráfego de dados se mostram essenciais. Importantes se tornam as circunstâncias responsáveis por garantir esta estruturação, cujo resultado depende do encadeamento de diversas ações pregressas.

Assim, o envolvimento de múltiplas organizações - associadas a partir de suas ações - colabora na 
constituição desta dimensão do contemporâneo.

Apreender o processo no qual as mídias se reconfiguram demanda uma discussão sobre a dinâmica da vida social, em seu processo contínuo. Trata-se de uma proposta que recorre à teoria da estruturação (GIDDENS, 1979), em sua expectativa de superar as dualidades contidas em abordagens exclusivamente sincrônicas ou diacrônicas. Apropriar esta orientação permite contribuir no debate sobre a constituição de sistemas de comunicação nos quais a transmissão ocorre a partir de redes digitais; a operação através de softwares; a associação entre equipamentos possíveis de oferecer mobilidade; todos estes traços mostram progressivamente a sua dimensão.

Entre os personagens cuja agência se mostra relevante se encontra a Telmex. Operação de telecomunicações com origem no México, inicia suas atividades no Brasil entre 2000 e 2002, expandindo sua atuação na América Latina. Ensaiando, em nosso país, com telefonia móvel, associa-se rapidamente ao audiovisual. Sua motivação reside em diversificar operações, decerto. Neste processo, estrutura recursos responsáveis por permitir a convergência entre telefonia fixa e móvel; internet em alta velocidade; televisão segmentada. No Brasil, estas ações encadeiam experiências posteriores, com impacto neste outro formato para a imagem. Observá-las significa apreender este momento de mudança, em um movimento diacrônico que envolve uma lógica sincrônica mais ampla.
Trata-se de um caso repleto de contradições, contudo. Para se conectar às atividades de audiovisual, a Telmex adquire, em 2004, uma participação na Net, operadora de televisão a cabo controlado pela Globo. A partir daí, fixa-se em convergir audiovisual e tráfego de dados. Organiza serviços como o Now, com foco em streaming, mas evitando as suas consequências efetivamente disruptivas. Mais importante, garante alguma sobrevida a um dos grupos anteriormente essenciais na televisão convencional: a Globo.

Para compreender estas múltiplas interações, este artigo se divide da seguinte forma. $\mathrm{Na}$ primeira parte, aborda-se a teoria utilizada: por um lado, a discussão sobre arqueologia da mídia (FOUCAULT, 1969; ZIELINSKI, 1994), identificando traços fundamentais à lógica que coordena este audiovisual na contemporaneidade; por outro, a ideia de estruturação, apropriando esta lógica abstrata frente a processos contextuais nos quais a agência procede. Ao considerar a importância da dimensão material, retoma-se na seção posterior os traços aos quais sistemas de complexos de audiovisual se referem, implícitos na constituição da televisão (KITTLER, 1999), mas, do mesmo modo, relevantes para a sua reestruturação. As duas próximas seções se debruçam sobre a investigação empírica relativa à participação da Telmex no Brasil: sua associação com a Globo e os desdobramentos da aliança entre ambas. 


\section{Orientações: arqueologia da mídia e teoria da estruturação}

Este artigo relaciona orientações distintas. Por um lado, congrega as ideias de arqueologia da mídia (FOUCAULT, 1969; PARIKKA, 2012), a fim de compreender a emergência dos formatos contemporâneos para os meios. Esta contribuição envolve uma abordagem ampla sobre a ordenação dos meios. A fim de apreender o seu caráter dinâmico, recorre-se à discussão de Giddens (1979) sobre a agência. Assim, atentase aos contextos nos quais os envolvidos lidam de forma hábil com os recursos disponíveis. Interpreta-se a noção de paradigma (DOSI, 1984; KUHN, 1962) como uma oportunidade de descrever este processo.

Uma abordagem arqueológica versa sobre a delimitação de objetos. Nos termos da discussão, estes objetos se referem à demarcação de elementos específicos da realidade, os quais, frente às circunstâncias disponíveis, diferenciamse e, assim, afirmam-se. Compreendem-se como objetos, por exemplo, os conhecimentos elaborados como as ciências do homem (FOUCAULT, 1966), instituindo positividade a certas formas de entendimento. Expandindo os estudos de Foucault sobre a ordenação destes saberes, a arqueologia da mídia se concentra nos meios em termos homólogos. Seu interesse consiste nas condições de possibilidade para, a partir das mídias, afirmar-se certa dimensão da percepção, em contraposição a outras.
Desse modo, 0 audiovisual delimita um regime de formas, possível através de recursos técnicos, de ações econômicas, de definições jurídicas, instituindo experiências particulares relativas à audição e à visão. No caso dos meios contemporâneos, as oportunidades de ver e ouvir se relacionam com a conexão capaz de reorganizar tempo e espaço. A partir da emergência deste objeto - as mídias -, ocorrem especificações particulares. Porém, a despeito das diversidades, a arqueologia identifica uma dada unidade, estabelecida a partir do discurso, conceito arqueológico que, como a ideia de objeto, apreende-se de forma particular.

Aqui, discurso diz respeito às práticas possíveis de delimitar estes objetos. Esta noção de discurso audiovisual não se confunde com uma análise semiológica sobre os discursos produzidos a partir dos audiovisuais. Nos termos de Foucault, refere-se a superfícies de emergência e a grades de especificação. Para o audiovisual, Zielinski (1994) identificou quatro especificações, resultados da diferenciação no interior do próprio objeto: as ilusões de movimento dos "pré-cinemas"; o cinema, a televisão e as assim chamadas audiovisões avançadas.

Apesar da qualidade de sua discussão sobre pré-cinema, cinema e televisão, a contribuição de Zielinski reside em delimitar as audiovisões avançadas. Este consiste em um tipo de audiovisual que rompe fronteiras entre as mídias, eliminando barreiras cujo exemplo mais dramático 
foi a dependência, para o cinema, da fotografia; para a televisão, dos pulsos elétricos. Recursos como a televisão de alta definição ou o cinema digital lidam com qualquer conteúdo através de uma mesma infraestrutura. Esta reorganização no interior do discurso audiovisual depende de software, instrumento essencial ao processo de digitalização.

Tema do trabalho de Chun (2011), indicou-se a especificidade do software enfatizando o seu caráter dúbio. Este instrumento de programação possui uma dimensão concreta, por operar como uma linguagem capaz de realizar tarefas. No entanto, sua operação se dá sempre de forma imaterial, frente à sua natureza como informação. 0 caráter sólido e evanescente transforma-se na contradição necessária de apreender. A natureza do software como código - um dado objetivo e inapreensível - define a natureza do digital: a possibilidade de, a partir de um processamento matemático, emular atividades das mais diversas.

Esta proposição permite inferir outras. 0s atributos concedidos às tecnologias de informação se inspiram em traços do próprio homem. Através de metáforas humanas, 0 software congrega a capacidade de lidar com informações. A partir daí, processa instruções sobre tarefas possíveis de realizar. Como consequência, desdobra ações concretas aptas a produzir efeitos. Todo este conjunto de atos se dá por programação. Entre as tarefas computáveis, encontra-se 0 armazenamento de dados, como um tipo de memória: em específico, uma memória em constante expansão. Novamente, contradições. Criar era uma possibilidade humana, mas criar por programação surge no limite do desumano; lembrar era coisa do homem, mas nossa memória depende de esquecimento, e não somente de assimilação.

0 software se encontra na fronteira entre 0 humanismo e sua dissolução. Capacidades até então exclusivas ao homem - em particular, a de lembrar - reorganizam-se, assemelhando-se ao código. A memória programável - humana, pois remonta à imagem de um atributo caro a esta criatura; desumana, por esgotar seu potencial ao confiscá-la para o software - se dá, na programação, por um movimento inexorável de acumulação de novas funcionalidades, congregando habilidades e reproduzindo-as através do software. Como marca, a programação indica a possibilidade de se estender em direção, no futuro, a tarefas ainda não emuladas no presente (MANOVICH, 2013).

\section{A constituição destes traços ocorre não como} consequência de uma estrutura, mas a partir de ações (GIDDENS, 1979). Condizente com a crítica ao estruturalismo, atenta-se ao contexto no qual se opera, enfatizando a prática e a agência. Concentra-se na reflexividade, abordando-a como irredutível a qualquer dimensão puramente sincrônica. Afirmam-se os traços típicos à organização do contemporâneo não como algo dado. A presença do software; a 
associação entre tecnologias de comunicação

e sistemas audiovisuais; a relação com características humanas redimensionadas; a expansão das capacidades de emulação por meio deste software, todas surgem como processos ordenados pela agência.

Nesta análise, tenta-se associar a ideia de estruturação com a discussão sobre paradigmas. Este conceito aborda o caráter cumulativo e não aleatório do desenvolvimento técnico, sem pressupor a sua linearidade. Paradigmas oferecem orientações sobre as possibilidades na exploração da técnica. Sua operacionalização ocorre a partir de trajetórias, operacionalizando tais paradigmas. Estes, por sua vez, pressupõem efeitos de exclusão, formato imposto a partir da adoção de certa técnica, cegando os envolvidos para outras possibilidades; e progresso relativo - ou seja, não absoluto -, como uma direção em relação a qual os traços da tecnologia se expandem, chegando, por fim, a uma fronteira intrínseca a cada paradigma (DOSI, 1984; KUHN, 1962). A exploração das oportunidades, sua expansão e seu esgotamento pressupõem, na perspectiva aqui proposta, a agência, tornando estes conceitos de paradigmas e trajetórias direções para apreender o processo de estruturação.

\section{A organização dos sistemas audiovisuais: paradigmas e trajetórias em operação}

A institucionalização das audiovisões avançadas e a adoção dos recursos decisivos para a trajetória em questão dependem da agência de operações de telecomunicações. A forma como instituem a infraestrutura necessária para a difusão de audiovisual adquire notável importância. Trata-se de uma segunda renovação dentro da mudança presenciada pelo audiovisual nas últimas décadas. Em diversos países - e também no Brasil - operadores de televisão segmentada a cabo e satélite haviam, durante os anos 1990 e 2000, estendido os limites das redes de televisão aberta (HOLT, 2011). Entretanto, a reorganização proporcionada pelo vínculo intensivo com tecnologias de informação irá depender não dos serviços com origem na televisão, mas de empreendimentos de telecomunicações e dos recursos que manuseiam.

Como decorrência da diversificação dos serviços de telecomunicações; da expansão dos sistemas de audiovisual para além dos meios aos quais se restringiram no passado; da operação por parte das organizações envolvidas em redes complexas; da ação de normas para a organização jurídica da atividade; devido a este conjunto amplo de questões, estas audiovisões avançadas radicalizam a trajetória pregressa do audiovisual. A presença de empreendimentos ligados a tráfego de dados; a distância que impõem em relação às atividades convencionais de audiovisual; a especificidade com a qual operam, consequência de dadas circunstâncias técnicas, econômicas e jurídicas: todas estas características definem a sua trajetória. Trata-se de mudanças que se inserem no 
paradigma para a exploração da televisão.

Desde os seus primórdios, esta televisão se constitui como um produto complexo. A adesão a tecnologias comparativamente mais sofisticadas não representa uma novidade. Já em sua origem, distingue-se de instrumentos mecânicos para elaborar representações. Sua operação envolve a produção de imagens através de correntes elétricas, gerando sinais distribuídos por circuitos eletrônicos. Pressuposto semelhante se percebeu no telégrafo, retirando da fala qualquer conexão com os estímulos físicos. Porém, uma diferença se mostra fundamental. Exemplo da complexidade inscrita neste tipo de audiovisual, a imagem codificada, ao contrário do texto, lida com dados em duas dimensões - vertical e horizontal -, cujo processamento em um eixo linear exemplifica a obrigatória sofisticação técnica.

A progressiva complexificação da televisão se insere nesta trajetória. A adição de cores, por exemplo, obrigava 0 aumento na capacidade para tráfego de dados. Sistemas mais complexos, dotados de outras funcionalidades, radicalizavam a demanda por sofisticação técnica. Exemplo se tornam os softwares imprescindíveis à operacionalização de HDTV (high-definition television). Associada às tecnologias de informação, a opção envolve algoritmos capazes de reduzir a quantidade de dados a transmitir, adequando-os às frequências toleráveis por sistemas convencionais para difusão. Como desdobramento, ampliar ainda mais as possibilidades de tráfego irá depender não de redes usuais de cabo, mas de tecnologias para fibra ótica, cuja operação envolve a transmissão a partir de luz, e não de eletricidade (KITTLER, 1999).

Estas redes de fibra radicalizam capacidades contidas no modelo pregresso, os sistemas de cabo coaxial ou os mistos coax-fibra (HFC, hybrid fiber $\operatorname{coax}$ ). Sua especificidade reside em ampliar tanto a velocidade quanto a capacidade de transmissão, eliminando interferências. HFC - importante na difusão da televisão a cabo, apropriado para as estruturas de DSL (digital subscriber line) utilizadas para internet em alta velocidade possui limites possíveis de superar somente a partir de fibras estendidas até residências (FTTH, fiber-to-the-home), proporcionando velocidades compatíveis com os serviços em ultra-alta definição, como 0 4K.

Como outrora, remete-se a técnicas complexas, inseridas em uma trajetória pautada pela expansão no uso de recursos imateriais para a apropriação da imagem. A solução envolve computação, e não a difusão por ondas eletromagnéticas. Em desdobramentos multivariados da representação numérica, estes instrumentos para 0 acesso ao audiovisual permitem romper, devido ao software, com sua apropriação linear no tempo. A quebra de sequencialidade envolve a expansão do audiovisual para aparelhos conectados - smart TVs, desktops, tablets, smartphones - permitindo 0 acesso a conteúdos em lugares variados. 
Por sua vez, o seu tráfego implica a funcionalidade adequada de redes físicas, cuja administração depende, entre outros, dos assim chamados CDNs (content delivery network), instrumento para racionalizar a localização de conteúdo em frações da rede mais próximas a usuários com intensa procura. Esta ferramenta transfere, por cópia, conteúdo com grande quantidade de acesso para um ponto próximo a localidades com intensa demanda. A operação destas redes obriga à automatização empreendida através de software.

Simultaneamente, estas experiências irão depender de codificação, adequando a imagem a diferentes plataformas (formatos específicos de tela demandam codificações distintas, por exemplo) e garantindo a sua operação em sistemas operacionais variados. Como parte da associação entre software e audiovisual, a programação obriga à padronização, garantindo funcionalidade aos serviços. Como resultado, dissociam-se os recursos para a distribuição de conteúdo daqueles utilizados para o seu uso. A circulação de material em complexos servidores se separa dos equipamentos domésticos, muito mais simples, que somente executam material. Para isto, tornase indispensável a computação em nuvem.

Ao mesmo tempo, necessita-se proteger este conteúdo, permitindo a sua exploração. Normatizar autorizações de uso significa instituir barreiras para a intensa possibilidade de cópia e circulação. Administra-se a segurança a partir de DRM (digital rights management), técnicas já consolidadas graças aos temores de "pirataria" (CHIDDIX et al., 2000; LEARN, 1988; MCADAMS, 2000; WILSON, 2008). Considerando este cenário pautado pela sofisticação das técnicas para a imagem, descreve-se na próxima seção em que termos se construíram historicamente as infraestruturas nas quais opera todo este conjunto de funcionalidades, percebendo como este processo se organiza através da ação, com ênfase à atuação da Telmex no país.

\section{Agência: Telmex e a estruturação de audiovisões avançadas no Brasil}

A Telmex consiste no sistema estatal de telecomunicações do México, privatizado em 1990. Sua negociação resulta na venda para a Carso, holding envolvida em atividades econômicas das mais diversas. Durante a segunda metade da década de 1990, a Telmex inicia sua expansão para outros países da América Latina, adquirindo operações de telecomunicações em El Salvador, Guatemala e Nicarágua, e serviços de telefonia móvel em nosso país (BALBONTÍN, 2005). No Brasil, sua atuação se inicia com a compra de empreendimentos que, durante a privatização do Sistema Telebrás, obtiveram outorgas para explorar telefonia móvel em banda B.

Garantir a sua presença em atividades de telecomunicações representa um investimento inicial em mobilidade, recurso importante em termos da mudança proporcionada pelas telecomunicações na experiência relativa a tempo 
e espaço. Mais relevante, associa-se à sua atuação futura com 0 audiovisual. Durante a privatização das telecomunicações no Brasil, definem-se dois tipos de serviços móveis. 0 primeiro se refere à assim chamada banda A. Até 1997, as empresas estaduais de telefonia que compunham o Sistema Telebrás possuíam, individualmente, suas operações de telefonia móvel. A fim de permitir sua privatização, separam-se essas empresas dos serviços fixos.

0 segundo tipo serão as atividades de banda $B$, responsáveis por permitir a entrada no setor de empreendimentos sem atuação prévia. Em ambos os casos, restringem-se estas outorgas a regiões delimitadas (NOVAES, 2000). Esta distribuição de concessões inicia a atuação, no Brasil, de operações internacionais de telecomunicações. A Telmex não se encontra entre elas. Suas aquisições futuras viabilizam os relacionamentos posteriormente encadeados: a associação com a Globo a fim de participar no audiovisual; a diversificação em atividades de telecomunicações dotadas de maior complexidade; a expansão geográfica, passando a atuar em vasta

Quadro 1: Distribuição de concessões para banda B de telefonia móvel, 1997-1998

\begin{tabular}{|c|c|c|}
\hline Região & Estado & Nome \\
\hline $\mathbf{1}$ & $\mathrm{SP}$ (capital) & BCP \\
\hline $\mathbf{2}$ & $\mathrm{SP}$ (interior) & Tess \\
\hline $\mathbf{3}$ & $\mathrm{RJ}, \mathrm{ES}$ & ATL \\
\hline $\mathbf{4}$ & $\mathrm{MG}$ & Maxitel \\
\hline $\mathbf{5}$ & $\mathrm{PR}, \mathrm{SC}$ & Global Telecom \\
\hline $\mathbf{6}$ & $\mathrm{RS}$ & Telet \\
\hline $\mathbf{7}$ & $\mathrm{AC}, \mathrm{DF}, \mathrm{GO}, \mathrm{MT}, \mathrm{MS}, \mathrm{RO}, \mathrm{TO}$ & Americel \\
\hline $\mathbf{8}$ & $\mathrm{AM}, \mathrm{AP}, \mathrm{MA}, \mathrm{PA}, \mathrm{RR}$ & Inepar \\
\hline $\mathbf{9}$ & $\mathrm{BH}, \mathrm{SE}$ & Maxitel \\
\hline $\mathbf{1 0}$ & $\mathrm{AL}, \mathrm{CE}, \mathrm{PB}, \mathrm{PE}, \mathrm{PI}, \mathrm{RN}$ & BSE \\
\hline
\end{tabular}

Fonte: (ABDALLA, 2012)

extensão do território brasileiro. Nestas ações, operacionalizam-se as trajetórias de conexão.

A presença da Telmex em nosso país começa pela associação, em 08/2000, entre Bell Canada, SBC,
Telmex, numa aliança estratégica denominada Telecom Americas (COLITT; MANDEL-CAMPBELL, 2000). Rapidamente, este grupo compra participações em quatro operações de telefonia móvel: Americel e Telet, em 03/2001; Tess, 04/2001; 
ATL, em 06/2001 ("America Movil acquires ATL stake", 2001, "Telia conclui venda dos ativos na Tess para Telecom Americas", 2001; CORNILS, 2001).

Já durante 0 seu envolvimento em telefonia celular, identifica-se uma lógica diversas vezes repetida: a habilidade em conduzir aquisições das mais variadas. Em várias interações, a Telmex, ao comprar empreendimentos com autorizações para operar em banda B, contribui para redefinir o formato delimitado após a privatização. Por sua agência, consolida-se a atividade. As oportunidades residem em dívidas contraídas por outras operações, empresas abandonadas por seus antigos proprietários frente à incapacidade de arcar com os investimentos necessários.

Após estas aquisições, a associação relativa à Telecom Americas começa a se esgotar. Iniciamse, então, negociações diversas, resultando na saída de Bell Canada e SBC em 06/2002. A

Quadro 2: Distribuição de concessões para banda B de telefonia móvel, 1997-1998

\begin{tabular}{|c|c|c|c|c|}
\hline Telecom Americas & ago/00 & jul/01 & fev/02 & jun/02 \\
\hline Bell Canada & $44,3 \%$ & $41,7 \%$ & $38,1 \%$ & N/A \\
\hline Telmex & $44,3 \%$ & $45,5 \%$ & $44,3 \%$ & $93,3 \%$ \\
\hline SBC & $11,4 \%$ & $12,8 \%$ & $10,9 \%$ & N/A \\
\hline Fundo investidor & N/A & N/A & $6,7 \%$ & $6,7 \%$ \\
\hline TOTAL & $100 \%$ & $100 \%$ & $100 \%$ & $100 \%$ \\
\hline
\end{tabular}

Fonte: (CORNILS, 2002; "Fundo investe para ser sócio na Telecom Americas", 2002, "Mexican Cellphone Concern Expands", 2002).

Telmex, então, passa a controlar 93,3\% da Telecom Americas, concentrando a propriedade das operações de telefonia móvel ("BCI conclui venda de participação no Telecom Americas", 2002).

Compras posteriores, conduzidas unicamente pela Telmex, permitem 0 controle de outras empresas constituídas durante a distribuição de concessões para banda B: BSE, em 03/2003;
BCP, em 08/2003 ("Telecom Américas compra BSE e chega a 6 milhões de assinantes", 2003; VARGA, 2003). A mesma lógica opera durante a aquisição, em 10/2003, da AT\&T Latin America, voltada a tráfego de dados corporativos ("Slim's march", 2004). Estas ações retomam o formato já descrito: a liquidação de empreendimentos endividados, incapazes de prosseguir com suas atividades. 
Na diversificação da Telmex, relevante se torna a compra da Embratel em 04/2004. Trata-se da aquisição de uma operadora de longa distância, dotada de infraestrutura para operação internacional, distinta das aquisições anteriores em telefonia móvel (POSSEBON, 2009, p. 208). Sua próxima ação se torna associar esta estrutura de telefonia e uma operação de televisão segmentada. Ao mesmo tempo, este acordo permite a conexão com a Globo, construindo outra rede, que, neste caso, envolve acesso a conteúdo.

\section{Em direção ao audiovisual: a compra da Net e a organização de streaming}

A compra da Net, serviço de televisão a cabo controlado pela Globo, ocorre em 06/2004.

Motivações para a venda se tornam 0 endividamento do operador, assim como os sistemáticos prejuízos responsáveis por impedir seu investimento em expansão. Adequando-se às normas em vigor, a aquisição obedece aos limites impostos pela Lei do Cabo de 1995 à participação do capital estrangeiro, que impede o controle de uma operação de cabo por um empreendimento econômico internacional. Nesta outra empresa em rede (CASTELLS, 1996), de formato bastante particular, torna-se essencial compreender 0 peso dos temas jurídicos e suas implicações para os esforços da Telmex voltados a audiovisões avançadas no Brasil.

Trata-se de um modo de lidar com os impedimentos legais em vigor; de permitir a possibilidade de controle após a transformação destas normas; de conduzir o relacionamento com os órgãos reguladores até 0 limite de suas possibilidades. Garantir uma participação com direito a voto na Net resguarda, para a Globo, determinadas prerrogativas. A despeito de perder 0 controle sobre a operação, tenta manter influência sobre decisões específicas. No acordo firmado durante a venda, a Globo garante poder de veto em contratos de programação. Assim, sem 0 seu consentimento não se pode alterar as disposições para a aquisição de conteúdo, relevantes no que diz respeito aos canais Globosat. Envolvia, também, decisões sobre a compra de produção estrangeira e de conteúdo nacional.

Frente à possibilidade de se aprovar a Lei de Serviço de Acesso Condicionado (SeAC, Lei 12.485, de 09/2011), transformando as regras para participação estrangeira em operações segmentadas, a Telmex reinicia negociações relativas à propriedade da Net, na expectativa de fechar 0 controle da operadora. Entre 10/2010 e 11/2012, discute-se entre os sócios a distribuição acionária da Net. A decisão da Telmex em assumir o controle requer que se redefina a influência da Globo. No final, a Telmex termina como 0 controlador de praticamente todo o capital da Net, tendo de manter, todavia, o poder de decisão da Globo sobre programação.

Este acordo indica a última opção para uma organização remanescente à organização do broadcast no Brasil garantir a sua importância 
em audiovisões avançadas. 0 uso de lacunas na regulação se torna essencial. Em sua formulação, a Lei do SeAC dividia o setor em quatro atividades: produção, programação, distribuição, empacotamento. No texto, compreendem-se somente as atividades de distribuição como serviços de telecomunicações. Empreendimentos deste tipo, estipula a lei, não poderiam ser controlados por organizações de audiovisual. 0 acordo entre os sócios garantia, para a Globo, a participação no conselho da Net, mantendo poder de veto. Conforme a Resolução 101/99 da Agência Nacional de Telecomunicações (Anatel), este poder de veto equivale a controle, indicando que uma atividade voltada ao audiovisual estava a administrar uma operação de telecomunicações.

Porém, a decisão da Net era a de se apresentar não apenas como uma atividade de distribuição, mas também de empacotamento, opção viável segundo a norma. Esta definição como empacotador produz um resultado constrangedor. A Anatel poderia intervir somente em temas relativos a operações de telecomunicações. Logo, a deliberação sobre este caso teria de envolver duas agências. Seguindo a Lei do SeAC, cabe à Agência Nacional do Cinema (Ancine) regular sobre questões que extrapolem a distribuição.

Por fim, obriga-se a Globo de abandonar o controle relativo a tudo, menos ao tema de seu efetivo interesse. Uma solução perfeitamente adequada às normas garante um resultado contrário àquele que estas regras haviam sido criadas para produzir. No novo acordo entre os sócios, apenas circunstâncias bem delimitadas anulariam o poder de veto da Globo: a probabilidade da Net, ao abrir mão de conteúdo, beneficiar outra operadora com maior número de assinantes; de um produto demonstrar a possibilidade de ampliar as vendas de outro empreendimento em mais de $30 \%$; de certo material se tornar importante para outro empreendimento em atividades de video on demand, ou VOD (ZERBONE LOUREIRO, 2012; ZILLER DE ARAÚJO, 2004).

Aquisições prévias realizadas pela Telmex afirmaram 0 controle sobre atividades de telecomunicações e garantiram sua presença no Brasil. Haviam eliminado diversos envolvidos, mas a única operação que resiste à consolidação será aquela voltada à programação de audiovisual. 0 resultado engloba questões jurídicas, apropriandose de brechas específicas da norma legal, garantindo a manutenção de um recurso de extrema importância.

É neste cenário que se organizam as atividades em streaming no Brasil. A difusão desta tecnologia, aqui, ocorre como um capítulo de sua internacionalização. Depende de empreendimentos de telecomunicações e da adoção sistemática de triple play (associação entre telefonia, internet e audiovisual), perceptível, em termos concretos, na relação entre telecomunicações e audiovisual. No âmbito de um paradigma específico, o streaming adquire sentido quando considerado lado a lado a estas 
experiências pregressas. No entanto, no caso da Net a institucionalização de audiovisões avançadas atenta a recursos pontuais, limitados frente às possibilidades presentes.

Observar estas experiências permite perceber as primeiras tentativas de lidar com 0 formato. Outra vez, este cenário retoma a associação prévia entre Telmex e Globo. Em 04/2011, a Net inicia o Now, serviço de streaming distribuído através de sua infraestrutura de HFC já existente, confiando na venda individual de material. A Globosat, por sua vez, divulga sua operação em 05/2011, identificada como Muu, cujo nome muda em 05/2014 para Globosat Play. Permite, para assinantes prévios da Globosat, acesso a conteúdo pela internet, expandindo a distribuição de produções através de outro mecanismo (BARB0SA, 2011; "Serviço de TV everywhere da Globosat tem nome: Muu", 2011).

Em 08/2011, Globo e Net se envolvem num acordo que associa o conteúdo e a infraestrutura de Muu e Now. Permite, inicialmente, acesso exclusivo ao conteúdo Globosat, retomando a lógica prévia, presente nas atividades de televisão segmentada. Entretanto, este caso possui as suas especificidades. Rapidamente, Muu inicia seu licenciamento para outras infraestruturas, possibilitando o acesso a CTBC em 04/2012, oferecendo posteriormente estes serviços para Oi, GVT e Telefónica ("Serviço de vídeo online Muu chega aos assinantes da CTBC TV", 2012, "Vivo TV passa a oferecer o Globosat Play", 2014). No serviço de cabo, o conteúdo da Globo havia se tornado uma especificidade valiosa, cuja negociação se evitou tanto quanto possível. 0 streaming consiste na especialização de ambas em tarefas distintas, contudo.

A expansão das atividades envolve a elaboração de outros serviços. Em 04/2012, a Globosat anuncia, em formato de teste, um serviço pago nomeado Philos, canal de documentários sobre arte. Seu lançamento se estenderia até $07 / 2012$, oferecendo conteúdo exclusivo, não distribuído nas estruturas de cabo (FREDERIC0, 2012). Seguemse, então, versões, no Muu, de Telecine, Premiere FC, Receitas GNT, Combate. Em 05/2013, a Globosat anuncia a possibilidade de lançamento do BIS +, serviço que envolveu cerca de dois anos de negociações com gravadoras, entre elas, Sony Music e Universal Music (CORDEIRO, 2013).

A criação de aplicativos para os serviços de Telmex e Globo se inicia com o lançamento, em 09/2011, de uma versão do Muu para equipamentos Apple, e, em 01/2012, para 0 Android. Por sua vez, o Now lança seu aplicativo muito tempo depois, em 08/2014. Em específico, garante a aquisição de conteúdo por clientes que não assinam a Net, ampliando o circuito de distribuição previamente delimitado (CORDEIRO, 2014; "Globosat cria aplicativos do Muu para iPad e iPhone”, 2011, "Muu ganha aplicativo para Android", 2012). Na adoção destes recursos, organiza-se uma forma de acesso a audiovisual que se distancia das operações convencionais da televisão. 
Ao considerar a reordenação do audiovisual como um processo, torna-se importante compreender as possibilidades que se afirmam a partir da agência dos envolvidos. As opções da Telmex ilustram a adesão a VOD, com atenção tardia a aplicações, recurso decisivo ao audiovisual associado a software. Concentra-se mais na expansão das funcionalidades das estruturas já existentes, e menos em experimentos com possibilidades disruptivas.

Foca-se na expectativa de, enquanto possível, deixar para o futuro a exploração mais sistemática dos desdobramentos inscritos em técnicas como o FTTH, confiando na apropriação, até o limite, dos recursos já existentes, ao invés de ampliá-los. 0 mesmo ocorre com a atenção a conteúdo já amplamente conhecido, oferecido pela Globo.

\section{Conclusão}

Percebendo a organização da vida social - em específico, as experiências com os sentidos proporcionadas pelas mídias - como consequência de um processo contínuo, discutiuse aqui um grupo particular de interações.

Debateu-se 0 envolvimento de Globo e Telmex na institucionalização do streaming, parte das audiovisões avançadas. Interpretaram-se essas ações recorrendo à ideia de discurso audiovisual, pautada, nesta grade de especificação, pela relação entre audiovisual e tecnologias de informação. Arqueologicamente, identificou-se este caráter como um problema de dimensão abstrata, necessário de apreender em termos das interações concretas que 0 estrutura.
Trata-se de uma discussão sobre agência. No caso da Telmex, o resultado se torna, via interações que envolvem a relação entre telecomunicações e audiovisual, a garantia de conectividade. Resguarda a si a possibilidade de manter seu envolvimento com um criador de intensa importância, permitindo organizar a sua operação de streaming. Afirma-se, então, como parte de um processo global de renovação da imagem, adotando estas tecnologias e permitindo a construção de mecanismos que distribuem conteúdo tão somente a partir de tecnologias digitais. Sua construção lida com um paradigma e suas trajetórias. Num esforço de diálogo, esta ideia de trajetória permite apreender os processos contextuais a partir dos quais se dá a convergência entre, por um lado, as experiências de ver e ouvir, e, de outro, a programação.

Contudo, recursos de dados e de imagem se encontram ambos associados de modo conservador. Telmex e Globo parecem confiantes em prosseguir num ritmo que evite 0 desconhecido. Transferem para as redes de difusão que criam os mesmos conteúdos já consolidados, caros aos sistemas de cabo. Mundo afora, o streaming tem se pautado pelo investimento sistemático em material inédito. Guia-se pela diversificação, pela aventura e a exploração de possibilidades inauditas no que se refere à diversificação criativa. Aqui, investe-se em opções que mantêm uma lógica já esquadrinhada, adiando 0 esgotamento do passado. Garante-se, por um lado, a flexibilidade no acesso a conteúdo. 
Por outro, exercita-se uma mistura dois mundos: um que se encerra, outro que se desenvolve.

\section{Referências}

ABDALLA, Rodrigo. Reflexões Sobre o Modelo de Autorização de Radiofrequências no Brasil. Radar, Brasília, v. 19, nº 2, p. 35-45, 2012.

America Movil acquires ATL stake. RCR Wireless News, Austin, v. 20, n 24, p. 30, 11 jun. 2001.

BALBONTÍN, Patricio Rozas. Privatización, reestructuración industrial y prácticas regulatorias en el sector telecomunicaciones.

Santiago de Chile: Cepál, 2005.

BARBOSA, Ana Carolina. Net lança VOD em São Paulo neste mês. Teletime, São Paulo, 14 abr. 2011.

BCI conclui venda de participação no Telecom Americas. Teletime, São Paulo, 25 jul. 2002.

\section{CASTELLS, Manuel. The Rise of the Network}

Society. $2^{\mathrm{a}}$ ed. Malden: Wiley-Blackwell, 1996.

CHIDDIX, Jim et al. The potential for hybrid fibre coax technology. Info, Bingley, v. 2, nº 2, p. 123-129, 2000.

CHUN, Wendy. Programmed Visions. Cambridge: The MIT Press, 2011.

COLITT, Raymond; MANDEL-CAMPBELL, Andrea. Mr Slim set for growth in bid to join big boys. The Financial Times, London, 9 ago. 2000. p. 26.

CORDEIR0, Letícia. Globosat lança o + BIS, canal de shows on-demand. Teletime, São Paulo, 17 maio 2013. Net Now agora pode ser acessado pela Internet. Teletime, São Paulo, 5 ago. 2014.

CORNILS, Patrícia. Bell Canada compra Americel e Telet. Valor Econômico, São Paulo, 14 mar. 2001. Novo sócio na Telecom
Econômico, São Paulo, 14 fev. 2002.

DOSI, Giovanni. Mudança Técnica e Transformação

Industrial. Campinas: Editora Unicamp, 1984.

FOUCAULT, Michel. A arqueologia do saber. Rio de Janeiro: Forense Universitária, 1969.

As palavras e as coisas. São

Paulo: Martins Fontes, 1966.

FREDERIC0, Daniele. Globosat lança canal de VOD por assinatura. Teletime, São Paulo, 12 abr. 2012.

Fundo investe para ser sócio na Telecom Americas.

0 Estado de S. Paulo, São Paulo, 14 fev. 2002.

GIDDENS, Anthony. Central Problems in Social

Theory. Berkeley: University of California Press, 1979.

Globosat cria aplicativos do Muu para iPad e iPhone.

Tela Viva, São Paulo, 26 set. 2011.

HOLT, Jennifer. Empires of Entertainment. New Brunswick, NJ: Rutgers University Press, 2011.

KITTLER, Friedrich. Optical Media. Cambridge: Polity, 1999.

KUHN, Thomas. The Structure of Scientific

Revolutions. Chicago: University of Chicago Press, 1962.

LEARN, Larry. Networks: A Review of Their Technology, Architecture and Implementation. Library Hi Tech, Bingley, v. 6, n. 2, p. 19-49, 1988.

MANOVICH, Lev. Software Takes Command. New

York: Bloomsbury Academic, 2013.

MCADAMS, Alan K. The world in 2010: the all fibre scenario. Info, Bingley, v. 2, nº 2, p. 153-166, 2000.

Mexican Cellphone Concern Expands. The New York

Times, New York, 4 jun. 2002. p. W1.

Muu ganha aplicativo para Android. Tela Viva, São Paulo, 2 jan. 2012.

NOVAES, Ana. A Privatização do Setor de 
Telecomunicações no Brasil. Rio de Janeiro: BNDES, 2000.

PARIKKA, Jussi. What is Media Archaeology? Cambridge: Polity, 2012.

POSSEBON, Samuel. TV por assinatura: 20 anos de evolução. São Paulo: ABTA, 2009.

Serviço de TV everywhere da Globosat tem nome: Muu. Teletime, São Paulo, 26 maio 2011.

Serviço de vídeo online Muu chega aos assinantes da CTBC TV. Teletime, São Paulo, 12 abr. 2012.

Slim's march. Latin Trade, Miami, v. 12, n. 6, p. 11, jun. 2004.

Telecom Americas compra BSE e chega a 6 milhões de assinantes. Teletime, São Paulo, 5 mar. 2003.

Telia conclui venda dos ativos na Tess para Telecom Americas. Valor Econômico, São Paulo, 10 abr. 2001.

VARGA, László. América Móvil compra BCP por US\$ 625 mi. Folha de S. Paulo, São Paulo, 30 ago. 2003.

Vivo TV passa a oferecer o Globosat Play. Teletime, São Paulo, 6 jun. 2014.

WILSON, Carol. Diverting the data flood. Telephony, Chicago, $1^{0}$ nov. 2008.

ZERBONE LOUREIR0, Rodrigo. Análise $\mathbf{n}^{0}$ 046/2012-GCRZ. Brasília: Anatel, 23 jan. 2012.

ZIELINSKI, Siegfried. Audiovisions: Cinema and Television as Entr'actes in History. Amsterdam: Amsterdam University Press, 1994.

ZILLER DE ARAÚJO, Pedro Jaime. Ato nº 48.245. Brasília: Anatel, 6 dez. 2004. 
The organization of streaming

in Brazil: Telmex, Globo and

the association between

telecommunications and audiovisual

\section{Abstract}

The article concentrates upon the agency presented at the structuration of streaming in Brazil, analyzing the interactions between two relevant characters in this scenario: Telmex and Globo. It investigates a particular dimension on the renovation of audiovisual in our country, with emphasis at their progressive association with information technologies. The institutionalization of this type of access to content demands the use of software, as a technique to diffuse material through the most diverse mobile devices. With emphasis at media archeology, this process will be observed as part of a broad scenario. Aiming to understand the logic of definition on this object, this audiovisual dependent on software will be related to the previous trajectory of television. This movement will be comprehended observing, as an empirical question, the bond between Telmex and Globo in their historical dimension, with attention to the way through which the network arrayed by both establishes the necessary infrastructure to formulate this fraction of the contemporary communication systems.

\section{Keywords}

Media archeology. Information and communications technology. Televisions studies

\section{La organización de streaming en Brasil: Telmex, Globo \\ e la asociación entre}

\section{telecomunicaciones e audiovisual}

\section{Resumen}

El artículo se concentra en la agencia presente en la estruturación de streaming en Brasil, analizando las interacciones entre dos personajes relevantes en este senario: Telmex y Globo. Investiga una dimensión particular en la renovación de audiovisual en nuestro país, con énfasis en su progresiva asociación con tecnologías de información. La institucionalización de este tipo de acceso a contenido demanda el uso de software, como una técnica para la difusión de material a los dispositivos móviles más diversos. Con énfasis en la arqueología de la media, se va observar este proceso como parte de un senario más largo. Con la expectativa de comprehender la lógica de definición de esto objeto, se va relacionar el audiovisual dependiente de software a la trayectoria de la televisión. Este movimiento se va comprender observando, como cuestión empírica, el eslabón entre Telmex y Globo en su dimensión histórica, con atención al modo como las redes ordenadas por ambos establecen la infraestructura necesaria para formular esta fracción de los sistemas contemporáneos de comunicación.

\section{Palabras clave}

Arqueología de la media. Tecnologías de la información e de la comunicación. Estúdios de televisión. 


\section{Expediente}

A revista E-Compós é a publicação científica em formato eletrônico da Associação Nacional dos Programas de Pós-Graduação em Comunicação (Compós). Lançada em 2004, tem como principal finalidade difundir a produção acadêmica de pesquisadores da área de Comunicação, inseridos em instituições do Brasil e do exterior.

\section{E-COMPÓS I www.e-compos.org.br I E-ISSN 1808-2599}

Revista da Associação Nacional dos Programas de Pós-Graduação em Comunicação.

Brasília, v.20, n.1, jan./abr. 2017.

A identificação das edições, a partir de 2008, passa a ser volume anual com três números.

Indexada por Latindex I www.latindex.unam.mx

\section{CONSELHO EDITORIAL}

Alda Cristina Silva da Costa, Universidade Federal do Pará, Brasil Alfredo Luiz Paes de Oliveira Suppia, Universidade Estadual de Campinas, Brasil Álvaro Larangeira, Universidade Tuiuti do Paraná, Brasil Ana Carolina D. Escosteguy, Pontifícia Universidade Católica do Rio Grande do Sul, Brasil Ana Regina Barros Rego Leal, Universidade Federal do Piauí, Brasil Ana Carolina Rocha Pessôa Temer, Universidade Federal de Goiás, Brasil Andrea França, Pontifícia Universidade Católica do Rio de Janeiro, Brasil André Luiz Martins Lemos, Universidade Federal da Bahia, Brasil Angela Cristina Salgueiro Marques, Faculdade Cásper Libero, Brasil Ângela Freire Prysthon, Universidade Federal de Pernambuco, Brasil Antonio Carlos Hohlfeldt, Pontifícia Universidade Católica do Rio Grande do Sul, Brasil Arthur Ituassu, Pontifícia Universidade Católica do Rio de Janeiro, Brasil Bruno Campanella, Universidade Federal Fluminense, Brasil Cláudio Novaes Pinto Coelho, Faculdade Cásper Líbero, Brasil Carlos Eduardo Franciscato, Universidade Federal de Sergipe, Brasil Denise Tavares da Silva, Universidade Federal Fluminense, Brasil Eduardo Vicente, Universidade de São Paulo, Brasil Eliza Bachega Casadei, Escola Superior de Propaganda e Marketing - SP, Brasil Elizabeth Nicolau Saad Corrêa, Universidade de São Paulo, Brasil Erick Felinto de Oliveira, Universidade do Estado do Rio de Janeiro, Brasil Erly Vieira Júnior, Universidade Federal do Espirito Santo, Brasil Francisco de Assis, FIAM-FAAM Centro Universitário, Brasil Francisco Elinaldo Teixeira, Universidade Estadual de Campinas, Brasil Frederico de Mello Brandão Tavares, Universidade Federal de Ouro Preto, Brasil Gabriela Reinaldo, Universidade Federal do Ceará, Brasil Gilson Vieira Monteiro, Universidade Federal do Amazonas, Brasil Gustavo Daudt Fischer, Universidade do Vale do Rio dos Sinos, Brasil Igor Sacramento, Fundação Oswaldo Cruz, Brasil Itania Maria Mota Gomes, Universidade Federal da Bahia, Brasil Jiani Adriana Bonin, Universidade do Vale do Rio dos Sinos, Brasil José Afonso da Silva Junior, Universidade Federal de Pernambuco, Brasil
José Luiz Aidar Prado, Pontifícia Universidade Católica de São Paulo, Brasil Juçara Gorski Brittes, Universidade Federal de Ouro Preto, Brasil Laura Loguercio Cánepa, Universidade Anhembi Morumbi, Brasil Liziane Soares Guazina, Universidade de Brasilia, Brasil Luíza Mônica Assis da Silva, Universidade Católica de Brasília, Brasil Maria Ataide Malcher, Universidade Federal do Pará, Brasil Maria Elisabete Antonioli, Escola Superior de Propaganda e Marketing - SP, Brasil Maria das Graças Pinto Coelho, Universidade Federal do Rio Grande do Norte, Brasil Marcel Vieira Barreto Silva, Universidade Federal da Paraíba, Brasil Marcia Tondato, Escola Superior de Propaganda e Marketing, Brasil Marli Santos, Universidade Metodista de São Paulo, Brasil Márcio Souza Gonçalves, Universidade do Estado do Rio de Janeiro, Brasil Mauricio Mario Monteiro, Universidade Anhembi Morumbi, Brasil Mauricio Ribeiro da Silva, Universidade Paulista, Brasil Mauro de Souza Ventura, Universidade Estadual Paulista, Brasil Mayka Castellano, Universidade Federal Fluminense, Brasil Micael Maiolino Herschmann, Universidade Federal do Rio de Janeiro, Brasil Mozahir Salomão Bruck, Pontifícia Universidade Católica de Minas Gerais, Brasil Nísia Martins Rosario, Universidade Federal do Rio Grande do Sul, Brasil Potiguara Mendes Silveira Jr, Universidade Federal de Juiz de Fora, Brasil Raquel Ritter Longhi, Universidade Federal de Santa Catarina, Brasil Regiane Regina Ribeiro, Universidade Federal do Paraná, Brasil Roberto Elísio dos Santos, Universidade Municipal de São Caetano do Sul, Brasil Rodolfo Rorato Londero, Universidade Estadual de Londrina, Brasil Sérgio Luiz Gadini, Universidade Estadual de Ponta Grossa, Brasil Simone Maria Andrade Pereira de Sá, Universidade Federal Fluminense, Brasil Simone Maria Rocha, Universidade Federal de Minas Gerais, Brasil Suzana Reck Miranda, Universidade Federal de São Carlos, Brasil Tarcyanie Cajueiro Santos, Universidade de Sorocaba, Brasil Tatiana Oliveira Siciliano, Pontifícia Universidade Católica do Rio de Janeiro, Brasil Veneza Mayora Ronsini, Universidade Federal de Santa Maria, Brasil

\section{CONSELHO CIENTÍFICO}

Cristiane Freitas Gutfreind, Pontifícia Universidade Católica do Rio Grande do Sul, Brasil Eduardo Morettin, Universidade de São Paulo, Brasil

Felipe Costa Trotta, Universidade Federal Fluminense, Brasil Irene de Araújo Machado, Universidade de São Paulo, Brasil

\section{COMISSÃO EDITORIAL}

Eduardo Antonio de Jesus, Universidade Federal de Minas Gerais, Brasil Marco Antonio Roxo da Silva, Universidade Federal Fluminense, Brasil Osmar Gonçalves dos Reis Filho, Universidade Federal do Ceará, Brasil

\section{CONSULTORES AD HOC}

Kelly C. de Souza Prudencio, Universidade Federal do Paraná, Brasil Francisco P. Jamil A. Marques, Universidade Federal do Paraná, Brasil Tiago Quiroga F. Neto, Universidade de Brasília, Brasil

\section{EQUIPE TÉCNICA}

ASSISTENTE EDITORIAL Márcio Zanetti Negrini REVISÃO DE TEXTOS Press Revisão EDITORAÇÃO ELETRÔNICA Roka Estúdio IMAGEM DE CAPA Silas de Paula

\section{COMPÓS I www.compos.org.br}

Associação Nacional dos Programas de Pós-Graduação em Comunicação

Presidente

Edson Fernando Dalmonte

Programa de Pós-Graduação em Comunicação

e Cultura Contemporânea - UFBA

edsondalmonte@uol.com.br

Vice-presidente

Cristiane Freitas Gutfreind

Programa de Pós-Graduação em Comunicação Social - PUC-RS cristianefreitas@pucrs.br

Secretário-Geral

Rogério Ferraraz

Programa de Pós-Graduação em Comunicação

Universidade Anhembi Morumbi

rogerioferraraz@anhembimorumbi.edu.br

CONTATO I revistaecompos@gmail.com 